Revista Interdisciplinaria de Humanidades, Educación, Ciencia y Tecnología

Año VII. Vol. VII. N¹. Edición Especial. 2021

Hecho el depósito de ley: pp201602FA4721

ISSN-L: 2542-3029; ISSN: 2610-802X

Universidad Nacional Experimental Francisco de Miranda (UNEFM). Santa Ana de Coro. Venezuela

Narcisa Liliana Durán-Ávila; Jenniffer Romina Cañarte-Murillo; José Napoleón Zambrano-Murillo; Carlos Alberto Ayón-Lucio

DOI $10.35381 / \mathrm{cm} . v 7 i 1.529$

\title{
Daño pulmonar causado por asbestos en trabajadores de la construcción
}

\section{Asbestos lung damage in construction workers}

\author{
Narcisa Liliana Durán-Ávila \\ narcisa.duran@unesum.edu.ec \\ Universidad Estatal del Sur de Manabí, Jipijapa \\ Ecuador \\ https://orcid.org/0000-0002-6088-0551 \\ Jenniffer Romina Cañarte-Murillo \\ jennifferomina123@gmail.com \\ Investigador Independiente \\ Ecuador \\ https://orcid.org/0000-0002-4523-8923 \\ José Napoleón Zambrano-Murillo \\ josezambrano1209@gmail.com \\ Centro Clínico Quirúrgico Hospital del Día, Jipijapa \\ Ecuador \\ https://orcid.org/0000-0003-3025-3095 \\ Carlos Alberto Ayón-Lucio \\ carlos-ayon95@hotmail.com \\ Centro de Salud Andrés de Vera/Coordinación Zonal 4, Portoviejo \\ Ecuador \\ https://orcid.org/0000-0001-7343-270X
}

Recibido: 05 de mayo de 2021

Aprobado: 10 de agosto de 2021 


\title{
RESUMEN
}

El objetivo de la investigación es analizar el daño que provoca el asbesto en los pulmones; desarrollándose desde un diseño bibliográfico de tipo documental. Con base a información obtenida de Google académico, Scielo, Elsevier, OMS, ScienceDirect, Dialnet, Repositorios digitales, y otros; se realizó una revisión de un total de 20 artículos, de los últimos 10 año, sobre el daño pulmonar que causa el asbesto en las personas expuestas directamente a este. El pronóstico de la enfermedad depende de la cantidad de asbesto inhalado y de la duración a la exposición a este, teniendo en cuenta que no es una enfermedad reversible. Prohibir el uso de asbesto es la única manera de prevenir daños pulmonares. Se concluye que el uso de asbesto es uno de los peligros más contaminantes para la naturaleza y la salud humana a niveles mortales, por ende, debe haber un control sobre la utilización de este material.

Descriptores: Lesión Pulmonar; microfibrillas; epitelio. (Tomado del DeCS).

\begin{abstract}
The objective of the research is to analyze the damage that asbestos causes in the lungs; developing from a bibliographic design of a documentary type. Based on information obtained from academic Google, Scielo, Elsevier, WHO, ScienceDirect, Dialnet, Digital repositories, and others; A review was made of a total of 20 articles, from the last 10 years, on the lung damage caused by asbestos in people directly exposed to it. The prognosis of the disease depends on the amount of asbestos inhaled and the duration of exposure to it, taking into account that it is not a reversible disease. Banning the use of asbestos is the only way to prevent lung damage. It is concluded that the use of asbestos is one of the most polluting dangers for nature and human health at deadly levels, therefore, there must be a control over the use of this material.
\end{abstract}

Descriptors: Lung Injury; microfibrils; epithelium. (Source: DeCS). 


\section{INTRODUCCIÓN}

El asbesto es una fibra mineral que se encuentra en formaciones rocosas en la corteza terrestre. (1) Conforma un grupo de minerales que se usan como materia prima para la elaboración de productos manufacturados, principalmente en materiales de construcción. (2) Inadvertidamente, libera microfibrillas que terminamos inhalando. Las más pesadas, que son las de mayor tamaño y diámetro, impactarán en las vías nasales y la faringe. Las de tamaño intermedio serán detenidas en la tráquea y los bronquios, y se eliminarán por el sistema mucociliar. (3) Las menores de una micra llegan hasta los alvéolos, donde son atrapadas por los macrófagos, pudiendo ser eliminadas por vía linfática o causar efectos fibrosantes u oncogénicos (4) Murray, en 1907, realizó una autopsia a un trabajador expuesto a cableado de asbesto y halló en su pulmón extensas áreas de cicatrización. (5)

Se ha documentado ampliamente el daño ocasionado en humanos por la exposición asbesto: enfermedades pulmonares como la asbestosis (una forma de fibrosis pulmonar), placas, engrosamientos y derrames pleurales, y daños del ADN que llevan al desarrollo de cáncer de laringe y pulmón y de mesotelio. (6)

Factores de riesgo, es causada por la exposición a polvos, humo, ácidos fuertes y químicos que el aire puede transportar. (7) La constante exposición del trabajo en polvos de asbestos, talco, algodón y carbón en los sitios de trabajo es un factor que conlleva un gran riesgo. Las enfermedades asociadas son: bronquitis, neumoconiosis, silicosis y asbestosis. (8)

La capacidad del asbesto inhalado para producir asbestosis, cáncer de pulmón y mesotelioma tanto en humanos como en animales establecidos, la exposición al asbesto en un entorno ocupacional dentro de la comunidad en general se reconoce como peligros importantes. (9)

El cáncer de pulmón es una enfermedad con un período de latencia prolongado que a diferencia de otras patologías derivadas del asbesto no está relacionado con la magnitud ni con la duración de la exposición, para su diagnóstico basta con encontrar entre los antecedentes exposición laboral al asbesto, aunque a veces se pueda 
encontrar en el lavado broncoalveolar fibras de asbesto (fundamentalmente crisotilo) (10) (11)

A través del tiempo se han realizado estudios donde se reconoce a este como cancerígeno número 1 en los humanos. Datos a nivel global revelan que todos los años mueren como mínimo 90.000 personas de tumores del tracto respiratorio, mesotelioma y asbestosis debido a la exposición al asbesto. (12)

Actualmente hay cerca de 125 millones de personas expuestas al asbesto en su lugar de trabajo en el mundo. Se estima que a nivel global cada año mueren como mínimo 107.000 personas por cáncer de pulmón, mesotelioma y asbestosis debidos a la exposición ocupacional al asbesto. (13)

A tal respecto, según datos publicados por la Organización Mundial de la Salud (OMS), en el mundo mueren 318.000 personas al año por enfermedad pulmonar obstructiva crónica (EPOC) asociada a agentes ocupacionales, y se calcula que el asbesto es el causante de aproximadamente la mitad de las muertes por cáncer profesional hasta la fecha se ha logrado la prohibición en 54 países, y en otros, la reglamentación sobre su empleo. (14) (15)

A nivel mundial Rusia, Canadá, China, Zimbawe y Brasil son los principales productores de asbesto. No se conoce la producción o consumo actual de estas fibras en el Perú; sin embargo, se tiene datos del 2004 donde la importación de asbesto fue de 876518 toneladas. (16)

Como es de destacar Venezuela importó en el año 2004 aproximadamente 3.800 toneladas de asbesto, y en el 2005 se reportaron 12 empresas importadoras con alrededor de 600 trabajadores, de los cuales se calculó un 10\% con exposición al amianto, no solo siendo Venezuela si no también Colombia Brasil países en desarrollo la cual incentivan el uso de estos productos y exponen el riesgo de los trabajadores que manipulan estos materiales causante para la salud, en la actualidad se ha prohibido su restricción y evitando el número de casos contagios por el asbesto. 
A partir de lo anteriormente señalado se busca analizar desde una perspectiva teórica la utilización indiscriminada del asbesto y cómo repercute el impacto ambiental perjudicial para el ser humano.

\section{METODOLOGÍA}

El estudio se enmarca en un diseño bibliográfico de tipo documental. Para el análisis se tuvo en cuenta mínimo 20 bibliografías con investigaciones y puntos de vista que describen enfermedades de personas que trabajan en construcciones y otras que por motivos diferentes estuvieron expuestas.

La información fue obtenida de Google académico, Scielo, Elsiever, OMS, ScienceDirect, Dialnet, Repositorios digitales, y otros. Se realizó una búsqueda con las palabras claves como asbesto, daño pulmonar y asbestosis.

Los criterios de inclusión fueron artículos de 10 años anteriores, en los idiomas español e inglés, se eligieron los estudios realizados a las personas que trabajaron en construcciones y sus familiares. Al igual que personas que están expuestas indirectamente al asbesto por vivir cerca de las instalaciones de la planta de asbesto. Se excluyó artículos de más de 10 años y que no contienen información clara sobre el daño que produce el asbesto.

\section{RESULTADOS Y DISCUSIÓN}

Se revisó un total de 20 artículos sobre el daño pulmonar que causa el asbesto en las personas expuestas directamente a este. De los cuales se incluyeron 10 revisiones bibliográficas, 4 artículos científicos, 3 trabajos de titulación, 1 publicación de la OMS, 1 informe de investigación y 1 revista.

Los primeros reportes que se tienen sobre los efectos del asbesto en la salud datan de 1907, cuando Murray describió el caso de un trabajador expuesto a cableado de asbesto cuya autopsia presentaba extensas áreas de cicatrización pulmonar. Sin embargo, fue Cooke, quien en 1924 estableció la relación entre la patología que denominó fibrosis pulmonar y la exposición a asbesto. Desde entonces se ha 
demostrado que el asbesto produce diversos tipos de neoplasias, como son los cánceres de pulmón, pleura, peritoneo, laringe, esófago, estómago, colon, recto y riñón, y enfermedades fibrosantes como asbestosis pulmonar, fibrosis pleural y placas hialinas pleurales. (3)

El pronóstico de la enfermedad depende de la cantidad de asbesto inhalado y de la duración a la exposición a este, teniendo en cuenta que no es una enfermedad reversible y ni mucho menos curable; si la asbestosis desencadena la aparición de un mesotelioma maligno el pronóstico empeora significativamente. Dicho esto la importancia del conocimiento de la enfermedad radica en la prevención que se tenga de esta. (17)

Muchos de los casos de mesotelioma se han descrito en mujeres e hijos de trabajadores del asbesto, como consecuencia de exposición doméstica (al menos 376 casos), en oicinistas de la industria del asbesto, y en personas que viven en la vecindad de las minas de asbesto, como resultado de la contaminación del aire. También se han notificado casos de asbestosis en mujeres e hijos de trabajadores del asbesto. (8)

El mesotelioma es una neoplasia relacionada con el uso de asbesto. Se ha calculado que el riesgo de mesotelioma se incrementa en un 0,020\% por cada 1000 fibras de asbesto por gramo de tejido pulmonar.

La prevalencia de asbestosis en serie de Guipúzcoa, siendo la población de 717.832 habitantes, es de 2.23/100.000. Se reunieron un total de 55 pacientes: 54 hombres y una mujer. Todos ellos eran mayores de 55 años al diagnóstico, y de los mismos, 36 habían fallecido a la fecha de la recogida de datos. La exposición a asbesto ha sido ocupacional en al menos el $60 \%$ de los pacientes (para el otro $40 \%$ no se recogió en la historia clínica el tipo de exposición). (18)

Un caso específico de contaminación en Colombia es el de la señora Ana Cecilia Niño quien estuvo expuesta durante 17 años a este material que le causó cáncer. Ana Cecilia Niño Robles (Bogotá, 1975) aunque nació en Bogotá, vivió durante 17 años en 
el municipio de Sibaté, Cundinamarca. Allí su padres tenían una casa, muy cerca a las instalaciones de la planta de fibrocemento de la empresa Eternit. (1) La incidencia y mortalidad por mesotelioma pleural es mayor en el municipio de Sibaté con respecto a los municipios de la provincia de sabana occidente, La población masculina abarco el mayor número de casos. La mortalidad por otros cánceres de tracto respiratorio fue más significativa en los municipios de la provincia de sabana occidente en contraste con el municipio de Sibaté. La incidencia de tumores del tracto respiratorio fue mayor en el municipio de Sibaté con respecto a los municipios de la provincia de sabana occidente. (7)

Se han descrito casos de mesotelioma en personas expuestas a asbesto de origen natural o a minerales parecidos al asbesto presentes en el suelo en regiones de Turquía, Grecia, Chipre, Córcega, Sicilia, Nueva Caledonia, la provincia china de Yunnan y California. Aunque este último grupo no se vería protegido por las medidas de control de la producción y el uso de asbesto, los otros grupos sí se beneficiarían. En la revisión se seleccionaron 20 artículos, de los cuales 10 indicaban casos de personas expuestas al asbesto y las diversas enfermedades que este causa en los pulmones. Los otros artículos muestran información sobre la composición del asbesto y por los medios que este llega dentro del cuerpo.

Los resultados de este estudio exponen que el asbesto es un material altamente dañino para la salud de los trabajadores de la construcción, sus esposas, hijos y familiares, al igual personas expuestas indirectamente, que viven cerca de lugares donde se manipula este material. Los casos son a nivel mundial, pero cada uno depende del tiempo de exposición y cantidad de asbesto inhalado. Actualmente el 90\% del asbesto mundial proviene de China, India, Rusia y Brasil. (19)

La exposición prolongada a estas fibras puede hacer que se formen cicatrices en el tejido pulmonar y provocar falta de aire. Los pacientes pueden presentar síntomas 10 o 20 años después, como tos crónica y dificultad para respirar. Con un riesgo excesivo de cáncer de pulmón a niveles de exposición acumulativos tan bajos como 1,5 años de fibra/ml. (20) 
Las medidas de prevención son escasas, en algunos países se ha puesto medidas sobre el manejo de este material. Mientras que en otros se ha prohibido el total uso o manipulación del mismo. (6)

Cualquier tipo de exposición a este material causa enfermedades a nivel del tracto respiratorio. (3)

\section{CONCLUSIÓNES}

El asbesto es un material utilizado ampliamente en el mundo, cuyos efectos en la salud están comprobados. Hasta la fecha no se ha demostrado con certeza que exista un límite seguro de exposición en el que no se corra riesgo.

En Latinoamérica y países en vías de desarrollo sufren, los obreros encargados de la manipulación de los asbestos teniendo problemas pulmonares en muchos casos causando hasta cáncer, confinando a su portador ayuda de respiradores y en casos más extremos cáncer y la muerte por la manipulación de la misma.

La mejor prevención es establecer diferentes medidas de seguridad eficientes para lograr proteger altrabajador en forma efectiva. En general, puede llegarse a que en lugar de establecer estándares numéricos máximos se elimine su uso o se sustituya. Se debe legislar, prohibir y, sobre todo, hacer cumplir la ley para proteger la salud y la vida de los trabajadores. 


\section{REFERENCIAS}

1. Cano Orozco N, Chávez Zuluaga D. Un pulmón en juego explotación minera en campamento Antioquia [Licenciatura]. Universidad Católica de Pereida; 2018.

2. Chungandro Villacrés AB. Exposición ocupacional al asbesto y su relación con el mesotelioma pleural maligno. Repositorio Digital. 2020 Enero.

3. Marín Martínez B., Clavera I.. Asbestosis. Anales Sis San Navarra [Internet]. 2005; 28( Suppl 1 ): 37-44.

4. Moreira Macías L. Exposición al asbesto en trabajadores de la construcción y su relación con la salud pulmonar. Revista San Gregorio [Internet]. 2019 [citado 10 marzo 2021];(29). Disponible en: http://dx.doi.org/10.36097/rsan.v0i29.925

5. Accinelli Roberto Alfonso, López Lidia Marianella. El asbesto, una epidemia todavía por controlar. Gac Sanit [Internet]. 2017 Oct; 31 ( 5 ): 365-367. Epub 30-Nov-2020. https://dx.doi.org/10.1016/i.gaceta.2017.02.011

6. Peña $\mathrm{H}$, Bohórquez Armijos E. El asbesto como elemento perjudicial en el ser humano y de impacto ambiental negativo a nivel mundial. Revista Ciencias Pedagógicas e Innovación [Internet]. 2017 [citado 10 abril 2021];5(2). Disponible en: https://doi.org/10.26423/rcpi.v5i2.182

7. Sobradillo Peña V, Villate Navarro J, Lasso Manzano M, Diaz Pedreira A. Intercambios gaseosos en la asbestosis pulmonar. Archivos de Bronconeumología [Internet]. 1982 [citado 10 abril 2021];18(4):200-205. Disponible en: https://doi.org/10.1016/S0300-2896(15)32352-8

8. Arcentales C. Asbestosis y su incidencia en casos de bronquitis agudas en menores de 5 años del recinto el porvenir parroquia Pimocha cantón BabahoyoLos Ríos durante primer semestre 2017. Proyecto de Investigación. Babahoyo: Universidad Técnica de Babahoyo, Tecnología Médica; 2017.

9. Abú-Shams K., Pascal I.. Características, propiedades, patogenia y fuentes de exposición del asbesto. Anales Sis San Navarra [Internet]. 2005; 28 (Suppl 1): $7-11$. 
10. Burgos Díez P, Pozuelo León R. Prevención de riesgos laborales derivados de la exposición a amianto [Internet]. UVaDOC Repositorio Documental de la Universidad de Valladolid. 2017 [citado 10 marzo 2021]. Disponible en: https://uvadoc.uva.es/

11. Martíneza C, Monsó E, Queroa A. Enfermedades pleuropulmonares asociadas con la inhalación de asbesto. Una patología emergente. Archivos de Bronconeumología . 2013;4(40):166-177.

12. Barragán Alvarado MF, González Mayorga NK. Contaminación por asbesto y su relación con la incidencia de melotelioma y otros tumores malignos del tracto respiratorio en la población del municipio de Sibate Cundinamarca e el periodo de 2007 - 2015 [Internet]. Repositorio Institucional UDCA. 2017 [citado 10 marzo 2021]. Disponible en: https://repository.udca.edu.co/handle/11158/778

13. Asbesto crisotilo [Internet]. Organización Mundial de la Salud. 2015 [citado 10 abril 2021]. Disponible en: https://www.who.int/

14. Sánchez-Millán IM. Repositorio Digital. [Online].; 2016 [cited 2016 Diciembre 08. Available from: https://reunir.unir.net/handle/123456789/4545

15. Torres Guerrero JC, Torres Uribe AM, Páez Torres M. La hora cero del asbesto en Colombia. Divulgación Científica [Internet]. 2020 [citado 10 abril 2021];(3):108-113. Disponible en: https://revistas.urosario.edu.co/index.php/RDC/article/view/8891

16. Accinelli RA, López LM. Asbesto: la epidemia silenciosa. Acta Med Peru [Internet]. 2016Aug.30; 33(2):138. Available from: http://www.amp.cmp.org.pe/index.php/AMP/article/view/64

17. Escobar Agudelo SP, de la Cuesta Herrera S, Gómez Correa N, Gómez Ramírez MC, Maya Montoya J, Ramos Zapata S, Vásquez T EM. Asbestosis: epidemiología, prevención y tratamiento (Asbestosis: epidemiology, prevention and treatment) (Asbestose: epidemiologia, prevenção e tratamento). Rev CES Salud Pública [Internet]. 26 de octubre de 2012; 3(2):251-8. Disponible en: https://revistas.ces.edu.co/index.php/ces salud publica/article/view/21 
Revista Interdisciplinaria de Humanidades, Educación, Ciencia y Tecnología

Año VII. Vol. VII. N¹. Edición Especial. 2021

Hecho el depósito de ley: pp201602FA4721

ISSN-L: 2542-3029; ISSN: 2610-802X

Universidad Nacional Experimental Francisco de Miranda (UNEFM). Santa Ana de Coro. Venezuela

Narcisa Liliana Durán-Ávila; Jenniffer Romina Cañarte-Murillo; José Napoleón Zambrano-Murillo; Carlos Alberto Ayón-Lucio

18. Salegi Etxebeste I, Maldonado De Sasia A. Asbestosis. Estudio retrospectivo de una serie de casos de Guipúzcoa y revisión bibliográfica [Internet]. Universidad del País Vazco. 2021 [citado 10 abril 2021]. Disponible en: https://addi.ehu.es/handle/10810/30808

19. Delgado G. Diemen, Mercado Alfonso, Preciado S. María de Lourdes, Dávalos P. Gabriel, Delgado C. Ashley. Placas pleurales por inhalación de fibras de asbesto. Reporte de dos casos y revisión de literatura. Rev. chil. enferm. respir. [Internet]. 2020 Sep; 36( 3 ): 204-210.

20. Algranti E, Ramos-Bonilla JP, Terracini B, Santana VS, Comba P, Pasetto R, et al.. Prevention of Asbestos Exposure in Latin America within a Global Public Health Perspective. Annals of Global Health. 2019;85(1):49. DOI: http://doi.org/10.5334/aogh.2341 